\title{
Reliability Analysis of Wireless Real-Time Control Networks
}

\author{
Péter Horváth, Mark Yampolskiy, Yuan Xue, Xenofon Koutsoukos and Janos Sztipanovits \\ Institute for Software Integrated Systems \\ Vanderbilt University \\ Nashville, Tennessee, USA
}

\begin{abstract}
Probability of successful delivery under deadline constraints is one of the most important performance measures in a wireless real-time multihop control and sensor network. In this paper we approach the problem of determining the probability of successful packet delivery by calculating the per-link outage probability for different fading channel models. We provide easily computable results for the end-to-end reliability for two different physical layer designs. Furthermore, we show that incorporating physical layer information into routing and scheduling decisions can result in significant performance improvements when strict deadlines are imposed on the system.
\end{abstract}

Index Terms-fading channels, modulation schemes, crosslayer design, wireless real-time networks

\section{INTRODUCTION}

Determining the outage probability in wireless ad-hoc and sensor networks received considerable attention in the recent years. Optimizing the resource allocation to minimize the outage probability in wireless mesh networks is generally a hard and well-investigated problem, see e.g. [1]. Most of these studies focus on systems in which the performance is predominantly impaired by interference due to channel contention. However, time-critical applications, such as those in industrial multihop control networks, don't tolerate the uncertainty stemming from contention-based schemes. When designing networking technologies for such applications, system designers tend to adopt restrictive design choices to maximize the predictability of the system. For instance, WirelessHART, a widely used industrial wireless mesh networking technology [2], imposes strict limits on the allowed number of nodes, and employs centralized network management to design conflict-free node schedules to eliminate interference within the network. Other means to improve predictability include a non-adaptive modulation scheme and fixed-length, statically scheduled, non-shareable time slots.

These provisions come at the expense of low spectrum efficiency and limited network scalability. On the other hand, the above mentioned design restrictions render the analysis of these systems much more tractable than that of more general mesh networks. Virtually, the only important performance indicator is the probability of the end-to-end successful delivery of packets, within an application-imposed deadline. Maximizing the success rate within WirelessHART by routing optimizations and forwarding policy improvements has been extensively studied. For example, [3] proposes a joint routing and scheduling scheme under idealized settings, i.e., links are impaired with independent Bernoulli losses and only next-hop neighbors are considered for routing.

In this paper we go beyond simple statistical link loss models and strictly next-hop routing. Instead, we analyze the effect of network geometry and wireless propagation effects on the end-to-end outage behavior. In particular, we investigate the success probability for multihop mesh networks considering different path loss exponents and links impaired with different multipath fading processes. Measurements also confirm channel fading being the most significant cause of packet losses in industrial networks [4]. We show that the link outage-based approach accurately predicts the end-to-end reliability, and consideration of realistic transmission models leads to better insight into cross-layer interactions between physical layer parameters, link scheduling, and multipath routing in WirelessHART.

The main contributions of this paper can be summarized as follows. Motivated by the design choices within WirelessHART, we provide tools to determine the end-to-end reliability of such centralized multihop networks for realistic channel fading models. We calculate the outage probability over a single hop in a noise-limited random network with Nakagami- $m$ fading for path loss exponents of 2 and 4 . We also conduct a systematic assessment of the applicability of IEEE $802.15 .4 \mathrm{~g}$, a new amendment of IEEE 802.15.4, as a potential replacement physical layer (PHY) technology for wireless real-time networks. Our study shows that IEEE 802.15.4g improves spectrum efficiency while maintaining or improving transmission performance when compared to the original 802.15.4 physical layer standard (PHY) adopted by WirelessHART, and the outage analysis applies equally well to this PHY.

The paper is organized as follows. Section II presents the assumptions about the system. Section III summarizes relevant related work. In section IV we analyze the outage probability for a single link. In section $\mathrm{V}$ we connect the link outages to the end-to-end reliability and validate the approach using simulations. Finally, Section VI explores routing and scheduling design choices for different network deployments.

\section{SYSTEM MODEL}

We study the publishing problem a multi-hop wireless mesh network, in which nodes periodically generate packets that need to be transmitted, possibly over multiple hops, to one 
of the Access Points (APs). The latter forward the packets to a gateway node for further processing. The packets are associated with a deadline of $D$ fixed-length transmission slots and also associated with routing information based on a centrally pre-determined routing graph. Possibly, multiple independent routing paths are available and routing is not restricted to the next-hop upstream neighbors.

For every uni-directional traffic flow, the network is represented as a directed acyclic graph $G(V, E)$. Contrary to the assumptions in [3], link loss probabilities are characterized by the probability of link outage due to actual channel fading, i.e., the instantaneous signal-to-noise ratio being lower than the value necessary to transmit the packet data successfully.

The physical communication assumes half-duplex, fixedrate radios, and relies on a synchronized MAC protocol with fixed time slot length, which encompasses both the data transmission and the acknowledgement frame. The acknowledgement being short and also the wireless channel being reciprocal, we assume the packet loss is dominated by lost acknowledgements. The MAC employs slot-by-slot channel hopping over many channels. The transmission schedules are also pre-determined by a central network coordinator, ensuring that only one node is transmitting on a single carrier frequency in a given time slot, effectively eliminating interference within the system. We assume that the properties of the modulation scheme and/or some channel blacklisting mechanisms allow us to neglect external interference as well.

Every node transmits with a fixed transmit power $P_{\mathrm{Tx}}$. To determine the average received power, we apply the conventional distance-dependent path loss models with path loss exponent $N>0$. The average received power is given as

$$
P_{\mathrm{Rx}}=P_{\mathrm{Tx}} C_{N} / d^{N}
$$

where $d$ denotes the distance between the nodes and the intercept $C_{N}$ is a model parameter. We use the frequencynonselective independent block fading assumption, i.e., we assume the fading is constant during the transmission of a single packet, then changes independently to an subsequent fading state. The independent fading assumption is justified by virtue of the frequency hopping MAC assumption, rendering successive transmissions statistically independent unless the channel coherence bandwidth extends beyond several channels. We will return to this issue when presenting simulation results in Section V.

The received signal is then

$$
r(t)=\sqrt{\frac{P_{\mathrm{Tx}} C_{N}}{d^{N}}} s(t) h(t)+n(t),
$$

where $s(t)$ is the transmitted signal, $h(t)$ is the realization of the frequency-flat fading, and $n(t)$ is the additive white Gaussian noise.

\section{RELATED WORK}

The outage probability for random networks has been treated systematically if the links are impaired with interference due to channel contention and Rayleigh fading, see [5] and the references therein. The design of the frequency hopping, time synchronized mesh protocol, serving as the basis of WirelessHART, is described in more detail in [6]. Multipath routing in Poisson random networks has been studied in [7], where conditions for the existence of reliable paths between two nodes is studied. The link scheduling problem is investigated in [8] for unconstrained network topologies and bidirectional real-time traffic in more generality.

The 802.15.4 family of standards is being continuously amended with new PHYs for various specific purposes. Among these amendments, the recently published $802.15 .4 \mathrm{~g}$ 2012 [9] standard proposes a new PHY, termed "Multirate Orthogonal Frequency Division Multiplexing (MR-OFDM)", relying on multicarrier transmission. The techniques employed in this PHY make it a promising candidate for future real-time networks in challenging environments, offering a significantly better spectrum efficiency that the original 802.15.4 PHY offers at comparable throughputs.

In this paper, we focus on $802.15 .4 \mathrm{~g}$ option \#2 and evaluate side-by-side with 802.15 .4 as a potential alternative PHY. This option uses OFDM with 48 data subcarriers, and the nominal channel spacing is $800 \mathrm{kHz}$, which is by a factor of 6 less than in the original 802.15.4. It allows adaptive modulation and coding with up to 6 different modulation/coding scheme (MCS) combinations for each possible bandwidth option. Using option 2, QPSK modulation with rate 1/2 convolutional code (MCS3) yields to a data rate of $400 \mathrm{kbit} / \mathrm{s}$. MCS2 is essentially MCS3 with a 2-fold repetition code in over the subcarriers, yielding $200 \mathrm{kbit} / \mathrm{s}$ PHY data rate.

\section{RELIABILITY MODELING}

Our aim in this section is to obtain outage expressions for a single link within the network. The per-link outage combined with the link schedule provides us with the end-toend reliability for a certain flow within the network. Channel models applicable to indoor industrial scenarios in the literature generally assume Rician or Nakagami-m fading [4], [10]. We primarily focus on Nakagami- $m$ fading with fading parameter $m$ as it lends itself for analytic treatment for random networks, providing more insight. First we summarize known results for a deterministic link distance $d$, then turn our attention to random Poisson networks.

\section{A. Deterministic network layout}

A link $l$ in the network suffers an outage event during a scheduled time slot if the instantaneous signal-to-noise ratio (SNR) is below a certain threshold $\eta$ necessary for reliable communications, whose probability is given as

$$
P_{\text {out }}=\operatorname{Pr}[\mathrm{SNR}<\eta] \text {. }
$$

The PDF of the instantaneous received signal power in the Nakagami- $m$ fading channel is known to be

$f_{p}(\bar{P}, p)=\left(\frac{m}{\bar{P}}\right)^{m} \frac{p^{m-1}}{\Gamma(m)} \exp \left(-m \frac{p}{\bar{P}}\right), \quad p \geq 0, \quad m>0.5$, 
where $m$ is the parameter of the distribution and $\bar{P}$ is the average received power. The Nakagami- $m$ fading simplifies to Rayleigh fading for $m=1$, and approximates the Rician fading with Rice factor $K$ for the choice of the fading parameter

$$
m=\frac{(K+1)^{2}}{2 K+1},
$$

covering typical channels encountered in practice.

This outage probability for the Rician flat fading channel can be written as [11]

$$
P_{\text {out }}=\left[1-Q_{1}\left(\sqrt{2 K}, \sqrt{\frac{2(1+K)}{\overline{\mathrm{SNR}}} \eta}\right)\right],
$$

where $Q_{1}(\cdot, \cdot)$ is the Marcum Q-function, and $\overline{\mathrm{SNR}}$ is the average received signal-to-noise ratio, and $K$ is the Rician factor. Specifically, given the known link distances $d$ and the path loss model in (1), $\overline{\mathrm{SNR}}=P_{\mathrm{Tx}} C_{N} /\left(d^{N} P_{n}\right)$ with $P_{n}$ being the noise power within the receiver bandwidth.

For the Nakagami- $m$ fading we have [11]

$$
P_{\text {out }}=\frac{\Gamma(m)-\Gamma\left(m, \frac{m}{\mathrm{SNR}} \eta\right)}{\Gamma(m)},
$$

where $\Gamma(\cdot, \cdot)$ is the (upper) incomplete Gamma function.

\section{B. Random network layout}

Now we present novel results pertaining to random networks. For a Poisson random network with intensity $\lambda$, internode distances are also random. Taking into account the fading and path loss, the outage probability is given as

$$
P_{\text {out }}=\int_{0}^{\infty} \operatorname{Pr}\left[d^{N}>\frac{1}{\eta} \frac{p C_{N}}{P_{n}}\right] f_{p}\left(P_{\mathrm{Tx}}, p\right) \mathrm{d} p .
$$

We are able to provide closed-form outage expressions for specific path-loss exponent values, namely, for $N=2$ and $N=4$. These two values can be regarded as extremes in real propagation environments, although path losses slightly less than 2 have also been observed in industrial environments [10].

1) The $N=2$ case: If the nodes are randomly distributed in two dimensions according to a Poisson point process with intensity $\lambda$, the CDF of the squared distance to the $n$th nearest neighbor is known [12, Eq. (6)]. Thus,

$$
\operatorname{Pr}\left[d^{2}>\frac{1}{\eta} \frac{p C_{2}}{P_{n}}\right]=\frac{\Gamma\left(n, \lambda \pi \frac{C_{2} p}{\eta P_{n}}\right)}{\Gamma(n)} .
$$

Combining (2) and (6) with (5) results in an expression formally being a Laplace transform, thus we can use [13, Eq. (3.10.1.1)] to arrive at the expression for the outage probability towards the $n$th nearest neighbor:

$$
P_{\text {out }}=\frac{\Gamma(m+n) m^{m-1}}{\Gamma(m) \Gamma(n) a^{m}} \cdot{ }_{2} F_{1}\left(m, m+n ; m+1 ;-\frac{m}{a}\right),
$$

where ${ }_{2} F_{1}(\cdot, \cdot ; \cdot ; \cdot)$ is Gauss's hypergeometric function and

$$
a=\frac{P_{\mathrm{Tx}} C_{2} \lambda \pi}{4 \eta P_{n}}
$$

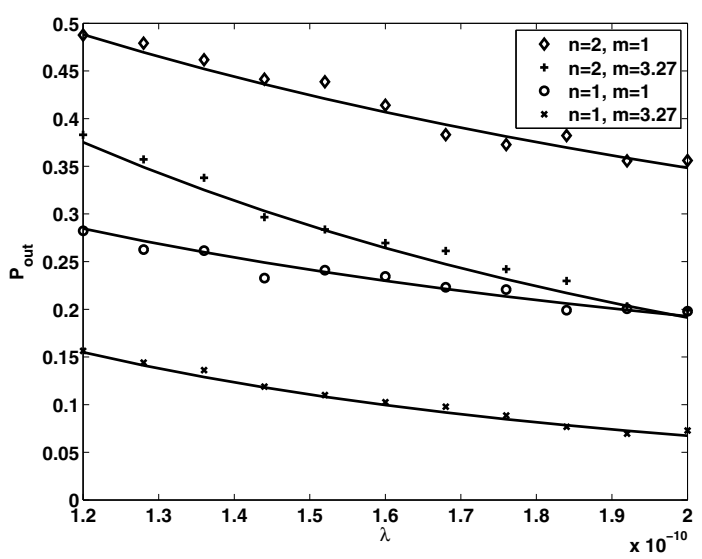

Fig. 1. Link outage probability for $N=2$ random networks with different node density, simulation vs. Eq. (7)

if the $n$th nearest neighbor is sought within an angular sector of $\pi / 2$ (routing towards a particular access point or field device), and

$$
a=\frac{P_{\mathrm{Tx}} C_{2} \lambda \pi}{\eta P_{n}},
$$

for forwarding to any node within the $2 \pi$ angle. Noting that ${ }_{2} F_{1}(1,2 ; 2 ; z)=1 /(1-z)$, Eq. (7) reduces to the well-known expression

$$
P_{\text {out }}=\frac{1}{1+a}
$$

for pure Rayleigh fading and $n=1$.

We have verified the validity of (7) using Monte Carlo simulations. For neighbors within the whole $2 \pi$ range, the agreement is demonstrated in Fig. 1.

2) The $N=4$ case: The distribution of $d^{2}$ to the $n$th nearest neighbor is given by [12] for a Poisson random network, and is also repeated here as (6). To determine the outage probability in the random network with path loss exponent $N=4$, one has to determine the distribution of $d^{4}$ to the $n$th nearest neighbor. Using the known distribution of $d^{2}$ from [12], one can show that appropriate transforms lead to the $\mathrm{CDF}$ of the fourth power of distance, which is

$$
F_{d^{4}}(r)=1-\frac{\Gamma(n, \lambda \pi \sqrt{r} / 4)}{\Gamma(n)}
$$

for neighbors within a 90-degree angular sector, and

$$
F_{d^{4}}(r)=1-\frac{\Gamma(n, \lambda \pi \sqrt{r})}{\Gamma(n)}
$$

otherwise.

Following the same lines as for the $N=2$ case, but using (9) instead of (6), we get

$$
\operatorname{Pr}\left[d^{4}>\frac{1}{\eta} \frac{p C_{4}}{P_{n}}\right]=\frac{\Gamma\left(n, \lambda \pi \sqrt{\frac{C_{4} p}{\eta P_{n}}}\right)}{\Gamma(n)},
$$

therefore evaluation of (5) with (2) and (10) becomes possible with [13, Eq. (3.10.1.7)]. However, instead of giving the extremely tedious analytic solution to (5) for a general $n$, 


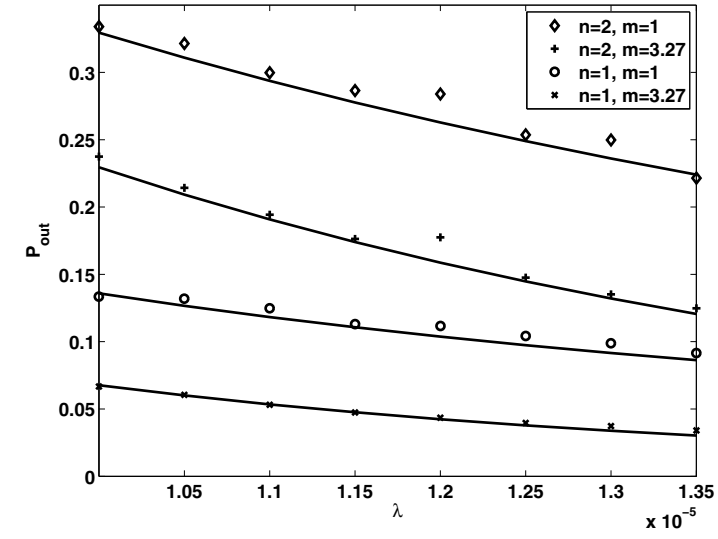

Fig. 2. Link outage probability for $N=4$ random networks with different node density, simulation vs. Eqs. (11) and (12)

we only present the outage probability for the two nearest neighbors. For $n=1$,

$$
P_{\text {out }, n=1}=\frac{\Gamma(2 m)}{2^{2 m-1} \Gamma(m)} U\left(m, \frac{1}{2}, \lambda^{2} \pi^{2} \frac{C_{4} P_{\mathrm{Tx}}}{4 m P_{n} \eta}\right),
$$

and for $n=2$,

$$
P_{\text {out }, n=2}=\frac{\Gamma(2 m)(2 m+1)}{2^{2 m-1} \Gamma(m)} U\left(m,-\frac{1}{2}, \lambda^{2} \pi^{2} \frac{C_{4} P_{\mathrm{Tx}}}{4 m P_{n} \eta}\right),
$$

where $U(\cdot, \cdot, \cdot)$ is the confluent hypergeometric function of the second kind. For $\pi / 2$ sector routing, $\pi$ needs to be replaced by $(\pi / 2)^{2}$ in (11) and (12).

The validity of (11) and (12) have also been verified using simulations, and the results are shown in Fig. 2.

\section{MOdel VALIDATION}

In this section we compare the reliability results obtained from the per-link reliability analysis with end-to-end reliability obtained from a detailed packet-level WirelessHART simulator. We focus on the known distance cases to verify the accuracy of the outage predictions.

\section{A. Link-level outage}

To determine the proper SNR threshold to be used for the outage probabilities we performed link-level simulations. This also allows us to compare the legacy 802.15.4 and the 802.15.4g OFDM PHY under realistic channel conditions. For the link-level evaluation we use three multipath fading models: the frequency-flat assumption, the IEEE 802.11n Channel Model D from [14] with $50 \mathrm{~ns}$ rms delay spread and a channel with a single exponential PDP, featuring a much higher rms delay spread than the 802.11n channels ("Exp" channel, 1000 ns delay spread).

Fig. 3. compares the link-level frame error rates for the legacy 802.15.4 PHY assuming a noncoherent correlation receiver, and the $802.15 .4 \mathrm{~g}$ option $2 \mathrm{MCS} 3$ receiver, respectively. Transmit power is fixed at $0 \mathrm{dBm}$, and the size of the payload

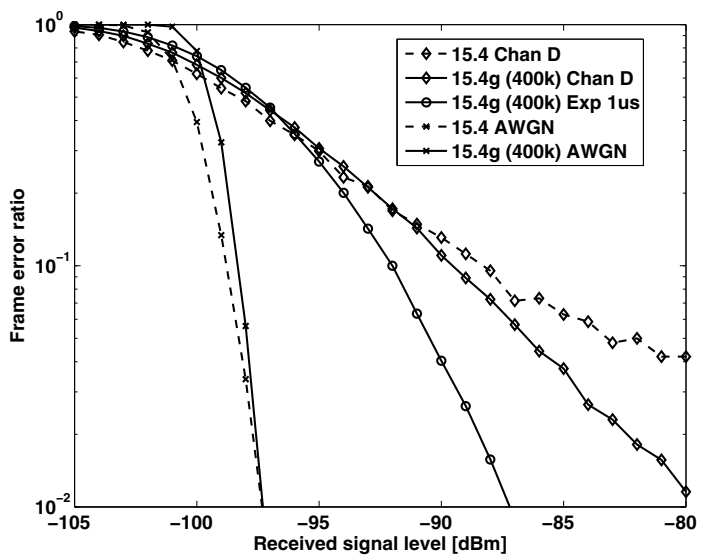

Fig. 3. Link-level frame error rate comparison between 802.15 .4 and 802.15.4g option 2 MCS 3

is 22 bytes. ${ }^{1}$ We can see that the non-fading (AWGN) performance is almost identical, whereas 802.15 .4 performance starts to deteriorate even for the rather benign Channel D Rayleigh fading model. The 802.15.4g receiver tolerates Channel D well, and, as expected, can gain significant performance from the more frequency-selective "Exp" channel, benefiting from the fact that in the more frequency-selective channel the subcarriers tend to fade more independently from each other. Therefore, the $802.15 .4 \mathrm{~g}$ offers higher data rate $(400 \mathrm{kbit} / \mathrm{s}$ vs. $250 \mathrm{kbit} / \mathrm{s})$ at 1/6th transmission bandwidth, and significantly better performance in dispersive channels.

\section{B. System-level validation}

We demonstrate that the analysis approach yields credible results on the system level, when end-to-end reliabilities are considered. To this end we simulate a regular network in which the nodes are placed on a lattice with variable inter-node distance, such as the one shown in Fig. 4. We compare the success probability obtained from the detailed packet-level WirelessHART simulator with the ones predicted based on the single-hop outage, (3). The simulator captures essential features of the scheduling and routing, and performs accurate bit-level evaluation of the MAC and PHY transmission procedures [15]. We present the success rate for packet transmissions from a field device to one of the access points over $H=5$ hops, when the hop distance is varied.

We assume a simple pre-determined slot schedule for both the primary and eventual redundant routing paths. If during the transmission period of $D$ time slots a link $l \in E$ is used $x_{l}$ times, the end-to-end reliability (probability of successful delivery with the deadline of $D$ time slots) can be expressed as [3]

$$
P_{\mathrm{succ}}=\prod\left(1-P_{\mathrm{out}, l}^{x_{l}}\right)
$$

\footnotetext{
${ }^{1}$ The evaluations are intentionally biased in favor of the legacy PHY: the IEEE 802.15.4 requirement for receiver sensitivity is $-85 \mathrm{dBm}$ at $\mathrm{FER}=1 \%$, whereas $802.15 .4 \mathrm{~g}$ requires only $-97 \mathrm{dBm}$ and $-100 \mathrm{dBm}$ for MCS2 and MCS3, respectively. We set the receiver noise figure so that the 802.15.4 receiver has the same sensitivity $(-97 \mathrm{dBm})$.
} 


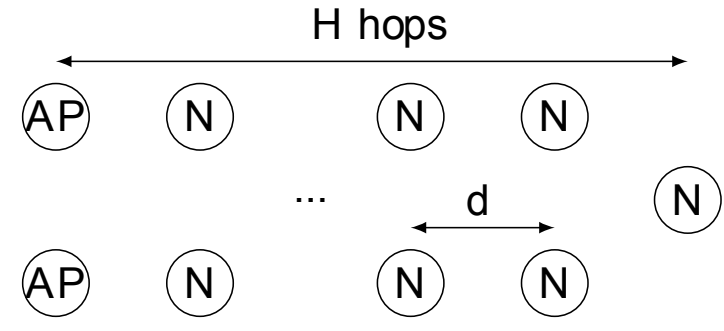

Fig. 4. Lattice layout

where $P_{\text {out }, l}$ is the probability of link outage for link $l$, determined by the path loss and fading distribution in our proposed analysis. This schedule is, according to [3], optimum for transmitting over a line of nodes, when the links are activated successively, from source to destination.

The path loss over a particular link is generated according to

$$
L(d B)=L_{0}\left(d_{0}\right)(d B)+10 N \log \left(d / d_{0}\right)
$$

where $d_{0}$ is a reference distance and $L_{0}\left(d_{0}\right)$ is the average path loss at the reference distance. We consider two propagation scenarios: pure free space for reference, and a realistic industrial indoor path loss law, derived from measurements [10]. The free space loss is characterized by $N=2$, at $2450 \mathrm{MHz}$ $L_{0}\left(d_{0}\right)=40.2 \mathrm{~dB}$ at a reference distance of $d_{0}=1$ meter. The measurement-based model is characterized by $N=2.48$ and $L_{0}(15 \mathrm{~m})=64.72 \mathrm{~dB}$. We run the simulations with pure Rayleigh fading, and with two different $K$ factors for the Rician channel.

Transmit power is fixed at $0 \mathrm{dBm}$, and onmidirectional antennas are assumed with a gain of $0 \mathrm{dBi}$. Due to the lack of advanced error control coding mechanisms in the WirelessHART 802.15.4 PHY, the dependence of the packet error rate on the SNR is not steep. Therefore the choice of the link outage threshold $\eta$ is somewhat arbitrary. We choose $\eta$ as the SNR where the packet error rate is $10 \%$ over nonfading AWGN channel for 38 byte packets (22 byte payload and 16 byte MAC overhead), which gives good agreement with the predicted outages. We also provide the comparison for the new 802.15.4g PHY option 2 MCS 3. The forward error control present in this PHY makes the frame error ratio dependence on the SNR steeper, hence choosing an appropriate SNR threshold is less ambiguous. The resulting success rates are shown in Fig. 5 for a 5 -hop line network using the best route without deadline constraint. The agreement between the simulation and theory is good both for small and large outages.

As a final remark, the single-hop outage analysis in Section IV relies on the assumption of independent fading realizations. The existing literature is not rich in providing channel characterization even for a single frequency in typical industrial environments, where multihop real-time networks often deployed. Especially the properties of the frequency-hopping channel can only be inferred from channel measurements conducted for broadband mobile communications, in quite different environments. Therefore one should assume that

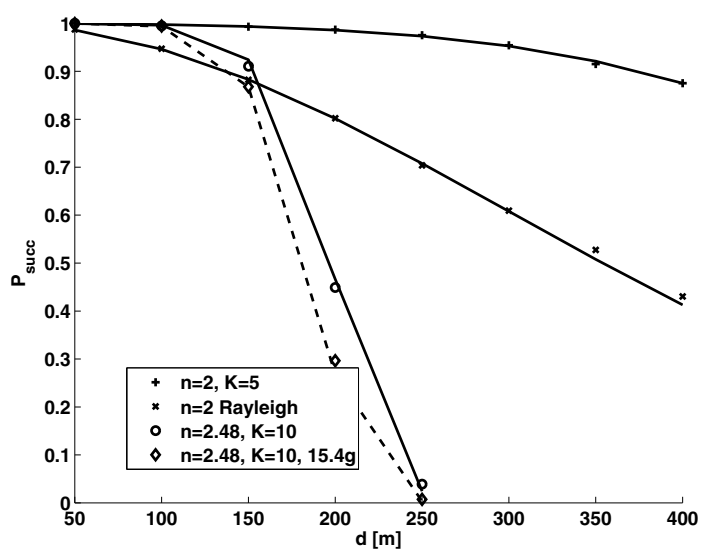

Fig. 5. End-to-end reliability for a 5-hop regular line network, best route (simulation vs. predicted success probability)

in certain environments the channel is highly correlated in frequency, the fading independence might not hold despite the channel hopping. To study a frequency-hopping PHY like the one in WirelessHART, we need to model the frequency correlation of the channel realistically.

For this purpose we have developed an analytical multichannel, multipath fading model, that allows tight control of temporal fading correlation. Through specification of a certain channel power delay profile, the model provides small-scale fading realizations at multiple carrier frequencies simultaneously. The frequency correlation between these realizations is determined by the temporal support of the prescribed power delay profile. The basis of the model is the channel simulation model presented in [16], in which the assumed power delay profile determines the frequency correlation, but the channel is still flat fading on a certain carrier frequency. We modified the simulation model presented in [16] to change the Doppler spectrum to Gaussian, which corresponds better to measurements of indoor channels.

Simulation results for the frequency-correlated channel are shown in Fig. 6. Again, a regular lattice of nodes is considered, and the 6-hop outage probability is considered under realistic propagation (including shadowing) and routing/link scheduling conditions. In particular, frequency-flat Rayleigh fading is generated with variable degree of frequency correlation over the entire $2.4 \mathrm{GHz}$ ISM band. The decreasing efficiency of the carrier frequency hopping with increasing fading frequency correlation causes systematic differences in the success rates. We enabled multipath routing and also allowed for one retransmission slot on every hop on the main routing path. Still, over highly frequency-correlated channels, the outage probability is unacceptably high. Under these circumstances multi-antenna techniques, such as simple delay diversity, could be the only means of mitigation. The 802.15.4g OFDM PHY lends itself naturally to such extensions, especially those MCS combinations that employ repetition coding across subcarriers. 


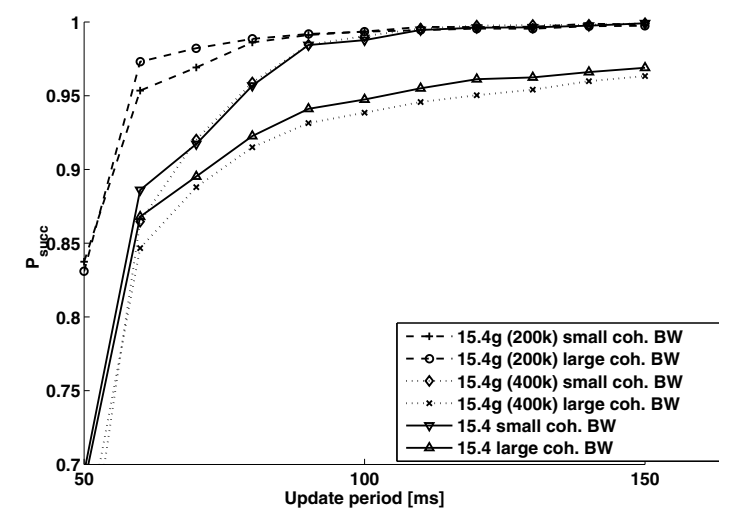

Fig. 6. Reliability for a 6-hop regular lattice over frequency-correlated flat fading channels

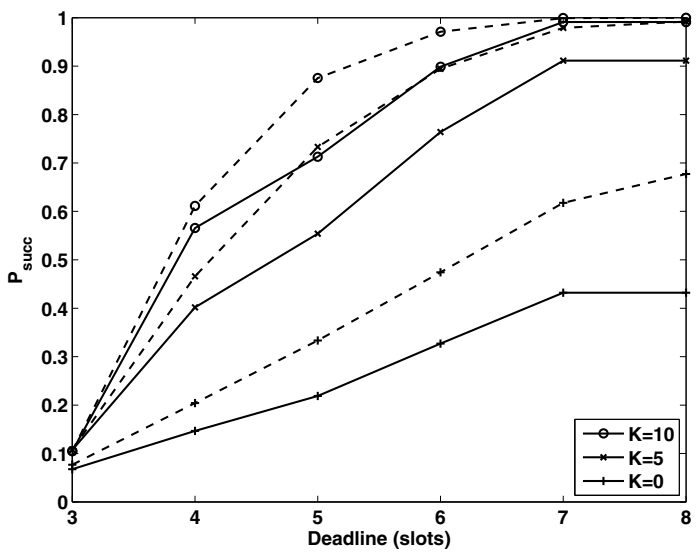

Fig. 7. Success rate versus Rician $K$ factor for the lattice network. Solid lines: single-line routing, dashed lines: redundant line routing

\section{CASE STUdies FOR SYSTEM DESIGN}

In this section, we show more numerical results for the success probability, based on the proposed framework, for different environments and deployment scenarios, in order to demonstrate the potential in cross-layer routing and scheduling design. First, a 7-hop regular lattice of nodes with internode distance $130 \mathrm{~m}$ and $N=2.48$ is being studied. The optimum routing for the given deadline is established by using dynamic programming [17], with the link weights set as $c_{l}=-\ln \left(1-P_{\text {out }}^{l}\right)$ with $P_{\text {out }}^{l}$ set to the respective perlink outage probability as calculated in Section IV, to obtain a routing decision that minimizes the end-to-end outage within the given deadline, eventually using non-adjacent hops as well. Every link along the route is activated only once.

Fig. 7 shows the succes rate versus the deadline for different Rician $K$-factors, single-line or redundant routing. Evidently, redundant routing offers a significant reliability advantage for small deadlines. This is in contrast with the findings of [3], which showed marginal advantages for homogeneous Bernoulli losses and strictly next-hop routing.

Figure 8 shows the effect of the hop length in a regular network. The distance between the source and the AP is kept

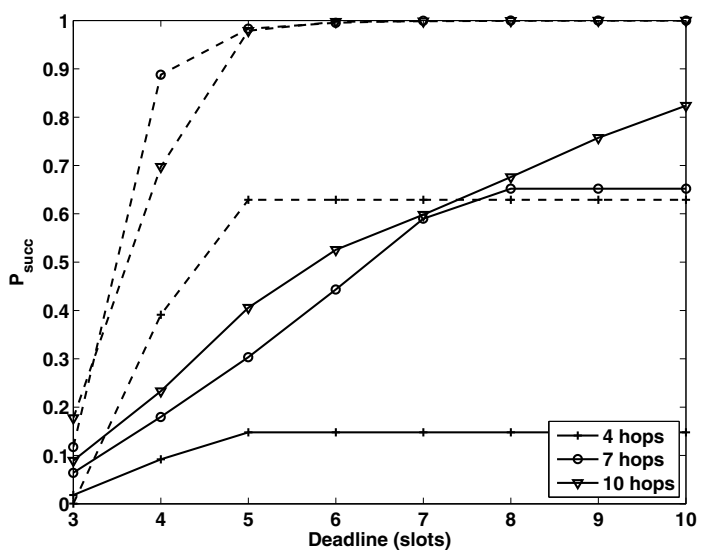

Fig. 8. Success rate versus hop length for the lattice network. Dashed lines: $K=10$, solid lines: $K=0 . N=2.48$

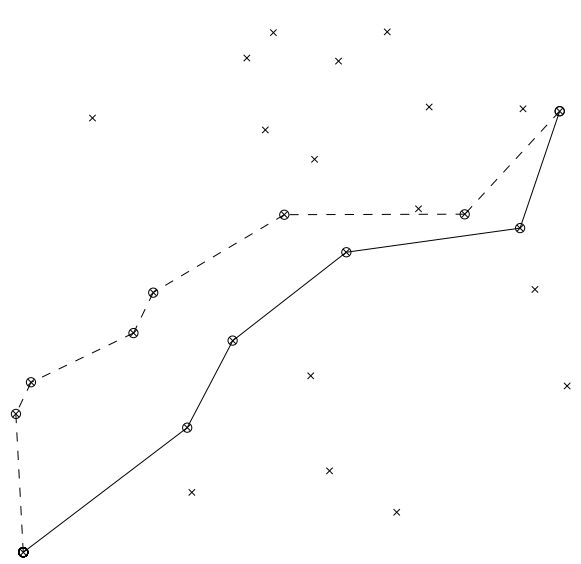

Fig. 9. Example Bernoulli layout; dots represent nodes, with primary (solid) and redundant (dashed) route

as $800 \mathrm{~m}$, but we consider diferent node densities $(4,7$, and 10 hops, respectively), between the source and AP, with redundant routing. It is seen that under less severe fading longer paths improve the performance for small deadlines, the 7 hop setting being superior to the 10 hop route. For longer deadlines this advantage obviously vanishes, the minimum outage route preferring a larger number of shorter, more reliable hops.

Similarly, we can assess the effectiveness of the redundant routing in a random network. We assume a scenario similar to the one depicted in Fig. 9, and investigate the $N=4$ case in which, due to high path losses, nearest neighbor routing is most likely for sparse deployments. It is reasonable to assume that the redundant route leads through different nodes than the the primary route, and the redundant route will favor nearest neighbors except for the first and last hop. Using (11) and (12) with (13) gives us the line reliabilities both for the primary and redundant route. When the AP is reachable in 5 hops, and the redundant routes are on average by one hop shorter than the primary ones, Fig. 10. shows the estimated gain in reliability of having an independent secondary route, for different fading statistics and deadlines (6,11 and 16 time slots, respectively). 


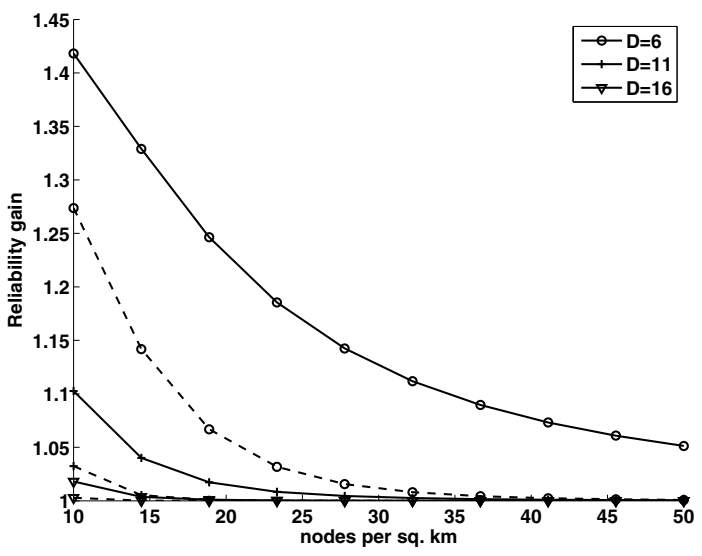

Fig. 10. Reliability gain of redundant routing for a 5-hop random network (solid lines: $m=1$, dashed lines: $m=3.27$ )

These findings confirm that redundant routing is advantageous in very sparse deployments, even if the fading is relatively benign, such as Nakagami- $m$ with a relatively high $m$-value. This advantage vanishes for less tight deadlines and denser deployments.

\section{CONCLUSIONS}

In this paper we approached the problem of predicting the reliability in real-time multihop frequency-hopping wireless networks under deadline constraints by analyzing the per-link outage probabilities with realistic wireless channel models. Simulation results support the applicability of our outagebased model to construct credible link loss models for future performance evaluation, instead of resorting to simple Bernoulli loss models. Closed-form expressions have been provided for the link outage under the assumption of certain path loss models that will help system designers evaluate the gains provided by multipath routing. We have shown that when deadlines are short compared to the minimum number of required hops, the reliability can be significantly improved by cross-layer routing decisions. Finally, the validation has also been extended IEEE 802.15.4g OFDM PHY, demonstrating its suitability for a state-of-art low complexity PHY for wireless real-time networks.

\section{ACKNOWLEDGEMENTS}

This work is supported in part by the National Science Foundation (CNS-1035655, CNS-1150169, CNS-1238959),
U.S. Army Research Office (ARO W911NF-10-1-0005) and Lockheed Martin. The views and conclusions contained herein are those of the authors and should not be interpreted as necessarily representing the official policies or endorsements, either expressed or implied, of the U.S. Government.

\section{REFERENCES}

[1] X. Shao, C. Hua, and A. Huang, "Outage probability based resource allocation in wireless mesh networks," in Proc. IEEE Global Telecommunications Conf. (GLOBECOM 2010), 2010, pp. 1-5.

[2] Industrial communication networks - Wireless communication network and communication profiles - WirelessHART, IEC Std. IEC 62591 Ed. 1.0, 2010.

[3] P. Soldati, H. Zhang, Z. Zou, and M. Johansson, "Optimal routing and scheduling of deadline-constrained traffic over lossy networks," in Proc. IEEE Global Telecommunications Conf. (GLOBECOM 2010), 2010, pp. $1-6$.

[4] D. Sexton, M. Mahony, M. Lapinski, and J. Werb, "Radio channel quality in industrial wireless sensor networks," in Sensors for Industry Conference, 2005, 2005, pp. 88-94.

[5] M. Haenggi, "Outage, local throughput, and capacity of random wireless networks," IEEE Trans. Wireless Commun., vol. 8, no. 8, pp. 4350-4359, 2009.

[6] K. S. J. Pister and L. Doherty, "TSMP: Time synchronized mesh protocol," in In Proceedings of the IASTED International Symposium on Distributed Sensor Networks (DSN08, 2008.

[7] C.-K. Chau, R. J. Gibbens, R. E. Hancock, and D. Towsley, "Robust multipath routing in large wireless networks," in Proc. IEEE INFOCOM, 2011, pp. 271-275.

[8] A. Saifullah, Y. Xu, C. Lu, and Y. Chen, "End-to-end delay analysis for fixed priority scheduling in WirelessHART networks," in Proc. 17th IEEE Real-Time and Embedded Technology and Applications Symp. (RTAS), 2011, pp. 13-22.

[9] IEEE 802.15.4g-2012: IEEE Standard for Local and Metropolitan Area Networks Part 15.4: Low Rate Wireless Personal Area Networks (LRWPANs) Amendment: Physical Layer (PHY) Specifications for Low Data Rate, Wireless, Smart Metering Utility Networks, IEEE Std., 2012.

[10] E. Tanghe, W. Joseph, L. Verloock, L. Martens, H. Capoen, K. Van Herwegen, and W. Vantomme, "The industrial indoor channel: large-scale and temporal fading at 900, 2400, and $5200 \mathrm{MHz}, "$ IEEE Trans. Wireless Commun., vol. 7, no. 7, pp. 2740-2751, 2008.

[11] M. K. Simon and M.-S. Alouini, Digital Communication over Fading Channels, 2nd ed. Wiley, 2006.

[12] M. Haenggi, "On distances in uniformly random networks," IEEE Trans. Inf. Theory, vol. 51, no. 10, pp. 3584-3586, 2005.

[13] A. P. Prudnikov, Y. A. Brychkov, and O. I. Marichev, Integrals and Series, Vol. 4: Direct Laplace Transforms. Gordon and Breach, 1992

[14] V. Erceg, L. Schumacher, and P. Kyritsi, "TGn channel models," IEEE 802.11-03/940r4, May 2004.

[15] P. Horvath, M. Yampolskiy, Y. Xue, X. D. Koutsoukos, and J. Sztipanovits, "An integrated system simulation approach for wireless networked control systems," in Proc. 5th Int Resilient Control Systems (ISRCS) Symp, 2012, pp. 118-123.

[16] C.-X. Wang, M. Pätzold, and Q. Yao, "Stochastic modeling and simulation of frequency-correlated wideband fading channels," IEEE Trans. Veh. Technol., vol. 56, no. 3, pp. 1050-1063, 2007.

[17] S. Dasgupta, C. Papadimitriou, and U. Vazirani, Algorithms. McGrawHill, 2006. 